\title{
Doppler Imaging of Stars with Roche-Geometry
}

\author{
Zs. Kövári ${ }^{1}$, J. Bartus ${ }^{2}$, K. Oláh ${ }^{1}$, K.G. Strassmeier ${ }^{2}$, \\ J.B. Rice ${ }^{3}$, M. Weber ${ }^{2}$ and E. Forgács-Dajka ${ }^{4}$ \\ ${ }^{1}$ Konkoly Observatory of the Hungarian Academy of Sciences \\ H-1525, Budapest, P.O. Box 67, Hungary \\ email: kovari, olah@konkoly.hu \\ ${ }^{2}$ Astrophysicalisches Institut Potsdam \\ An der Sternwarte 16, D-14482, Potsdam, Germany \\ email: jbartus, kstrassmeier, mweber@aip.de \\ ${ }^{3}$ Department of Physics \& Astronomy, Brandon University \\ Brandon, MB, R7A 6A9, Canada \\ email: rice@brandonU.ca \\ ${ }^{4}$ Department of Astronomy, Eötvös Loránd University \\ H-1518 Budapest, P.O. Box 32, Hungary \\ email: E.Forgacs-Dajka@astro.elte.hu
}

\begin{abstract}
Tests are carried out on retrieving Doppler maps from distorted stars in close binaries to estimate how Doppler imaging may be aliased by ellipticity. Maps obtained for the distorted shape are compared with the results of the simple spherical approximation, using real data of the RS CVn-type close binary star $\zeta$ Andromedae.
\end{abstract}

Keywords. techniques: spectroscopic, stars: activity, (stars:) binaries: eclipsing, stars: fundamental parameters (ellipticity, temperatures), stars: imaging, stars: individual ( $\zeta$ And)

\section{Introduction}

In our recent paper (Kővári et al. 2005) we presented the first Doppler images of the giant component of the close binary star $\zeta$ Andromedae (HD 4502). Since our Doppler imaging code TempMap (Rice, Wehlau \& Khokhlova 1989) did not take into account the distorted geometry, we were obliged to use a simple spherical approximation for the star, which, on the other hand, shows ellipticity effects in its photometric light curves (Hall 1990, and references therein). As a critical remark we noted that the first Doppler maps for $\zeta$ And with spherical approximation might be aliased by the distorted shape.

In this paper we carry out tests on retrieving Doppler maps from nonspherical stars distorted by tidal forces and gravitation in close binary systems (i.e., having Rochegeometry) to estimate the error introduced by the assumption of spherical shape. Finally, in Section 3 we turn back to our program star $\zeta$ And and taking into account the distorted shape we set a consistent model for further Doppler imaging (see our forthcoming paper by Kővári et al. 2006).

\section{Extending our DI code}

In our model Roche-geometry is approximated with a more handy 'cigar' shape, i.e., a rotation ellipsoid elongated towards the secondary component. The elliptical distortion parameter $\epsilon$ is defined as 


$$
\epsilon=1-\left(\frac{b}{a}\right)^{2}
$$

where $a$ and $b$ are the long and the short axes, respectively (see Figure 1). This is a purely geometrical approach because it does not account for the effect of gravitational darkening. On the other hand, the point-to-pole gravity ratio for $\epsilon=0.27$ (96\% nonsphericity, cf. Figure 3) is only 0.92 which, according to Zeipel's (1924) $T \propto g^{0.08}$ law converts to a point-to-pole temperature gradient of $\approx 30 \mathrm{~K}$, which is below the resolution capability of our data.
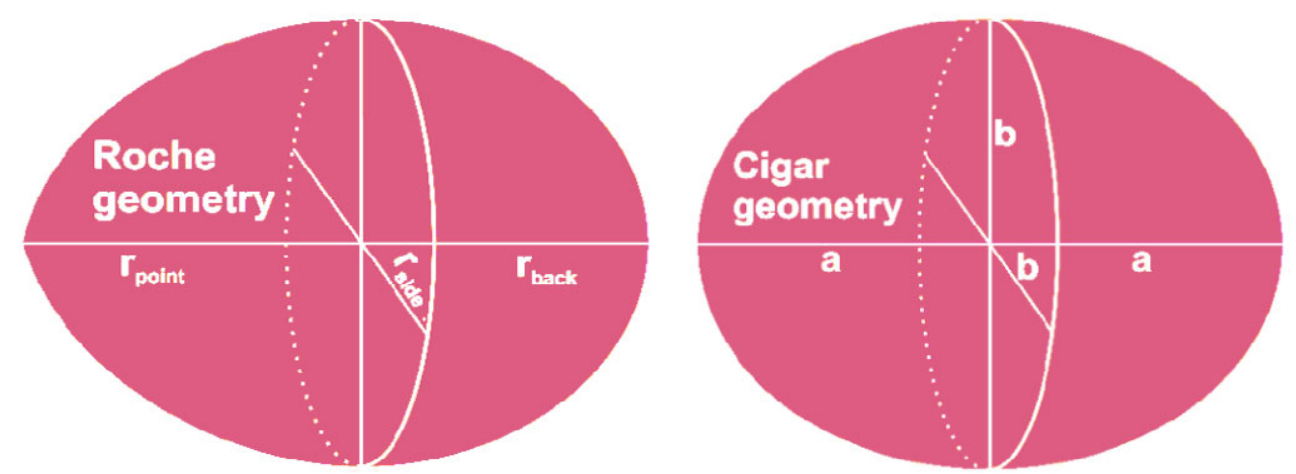

Figure 1. In our first order model Roche-geometry is approximated by a more handy 'cigar' shape.

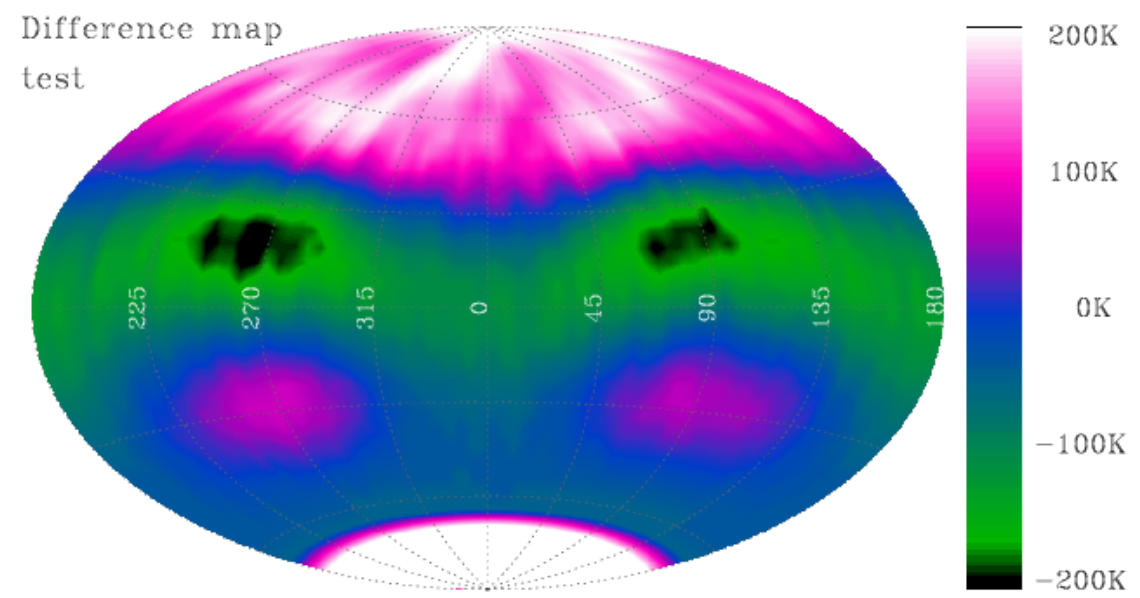

Figure 2. Test result of Doppler imaging a cigar shape star $(\epsilon=0.2)$. For making a series of artificial spectra we assumed homogeneous surface temperature $\left(T_{\text {eff }}=4600 \mathrm{~K}\right)$. When remodelling the spectra we assumed spherical shape. In the resulting Doppler map the original nonspherical shape is compensated by cooler and hotter patches with temperature differences of a few hundred Kelvins. Here we only plot the temperature difference map, i.e., the originally assumed homogeneous temperature distribution is subtracted from the resulting temperature map. The plot is presented in Aitoff projection with zero meridian at the centre, thus the 'back side' corresponds to phase value $\phi=0^{\circ}$, while 'point end' (facing the secondary) to $\phi=180^{\circ}$. 


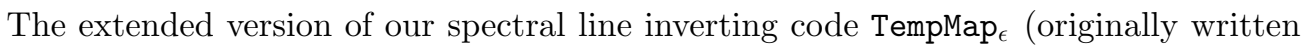
by Rice, Wehlau \& Khokhlova 1989) is then used to generate a series of synthetic spectra for a homogeneous surface tempetarure $\left(T_{\text {eff }}=4600 \mathrm{~K}\right)$ star with cigar shape $(\epsilon=0.2)$, otherwise we set the input parameters to fit the astrophysical data of $\zeta$ And (see Kövári et al. 2006).

We use these artificial data to see how temperature maps are affected by the application of a simplified spherical geometry: we make a Doppler image assuming a simple spherical shape, i.e., we set $\epsilon$ to zero. It is well seen in the resulting difference map (Figure 2) how the inversion tries to equalize the bad approximation of the geometry with cooler and hotter patches on the surface. This means that neglecting distortion can introduce an additional systematic error into the temperature inversion on the order of a few hundred degrees. Cooler areas concentrate at phases $\phi=90^{\circ}$ and $\phi=270^{\circ}$, i.e., when the cigar is at side-on view $\left(\phi=0^{\circ}\right.$ corresponds to the conjunction with the primary in front), however, hotter patches appear mainly around the pole representing a smaller but always visible contribution.

\section{The proof of the pudding}

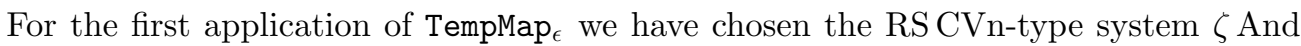
(HD 4502), which is a single-line spectroscopic binary with a distorted giant component reaching $80-100 \%$ of its Roche volume (Hall 1990). Spectral type is classified as K1 III (Strassmeier et al. 1993) with a possible F companion which is unseen. In the photometric light curves light variation due to the ellipticity effect dominates (see Kővári et al. 2006), however, traces of spot activity are also present (Strassmeier et al. 1989).

When performing Doppler Imaging several trial-and-error like iteration steps are made until one finds the best-fit model in the multidimensional parameter space. The most important parameters to be searched, among others, are: stellar inclination $i$, projected equatorial rotational velocity $v \sin i$, chemical abundances, turbulence parameters and, in

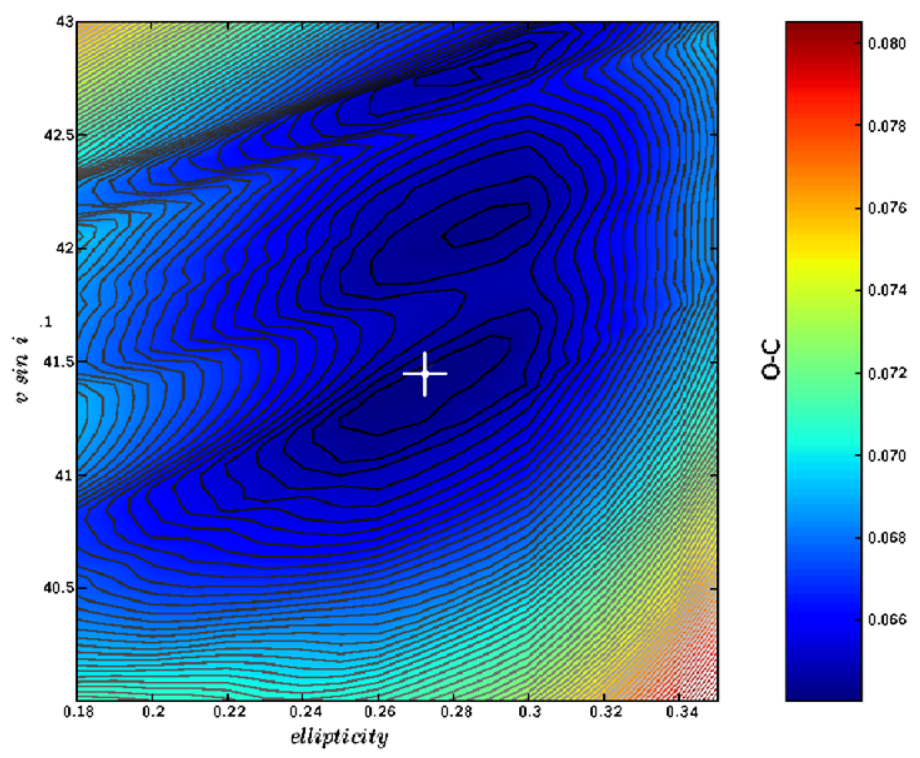

Figure 3. Parameter search with using the Doppler Imaging procedure. Plotted are the $\chi^{2}$ values over the $\epsilon-v \sin i$ cross-section of the parameter space. The minimum is marked with a white cross at $\epsilon=0.27$ and $v \sin i=41.4 \mathrm{~km} \mathrm{~s}^{-1}$. 

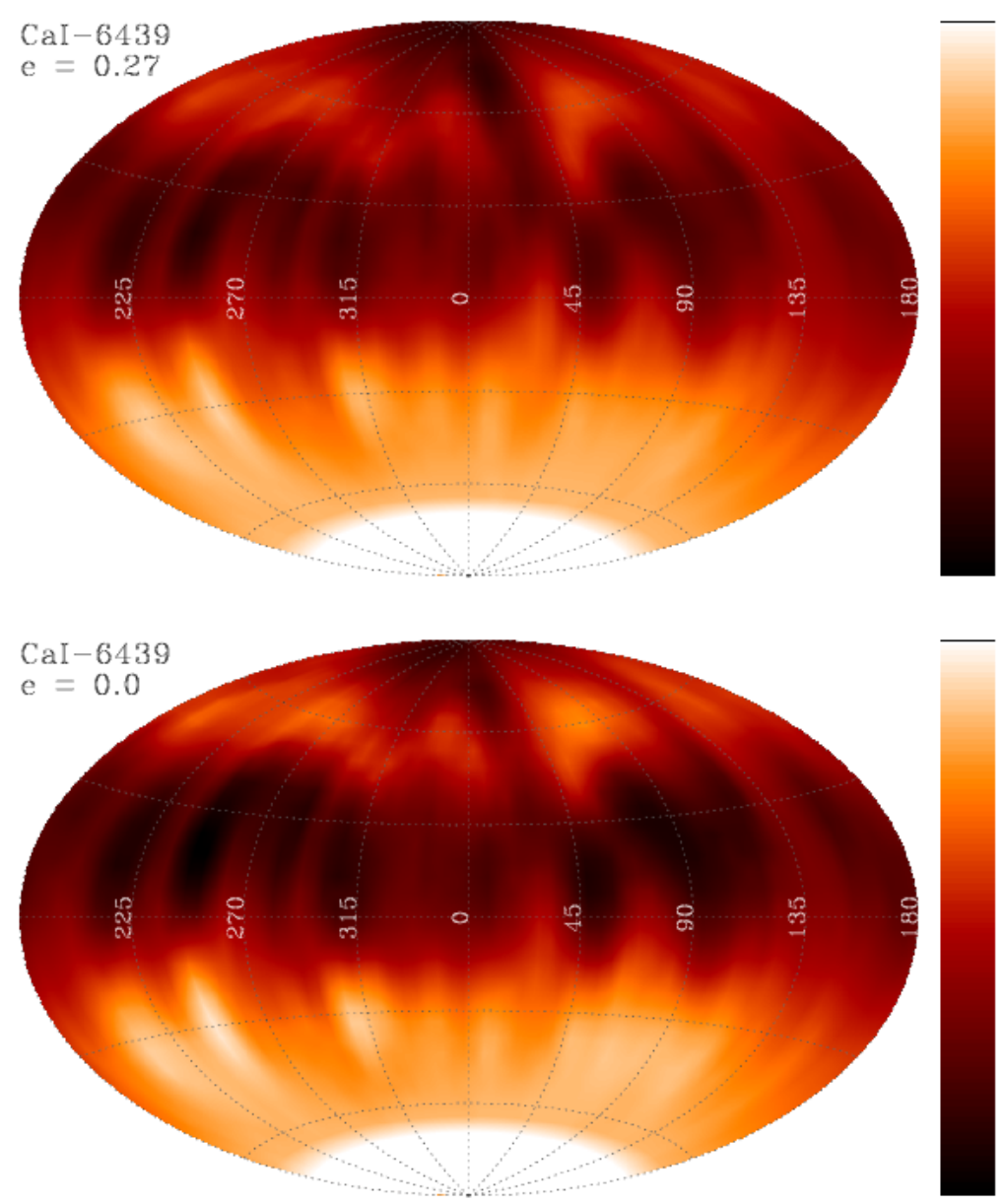

$4450 \mathrm{~K}$

$4100 \mathrm{~K}$

$3750 \mathrm{~K}$

$3400 \mathrm{~K}$

$4800 \mathrm{~K}$
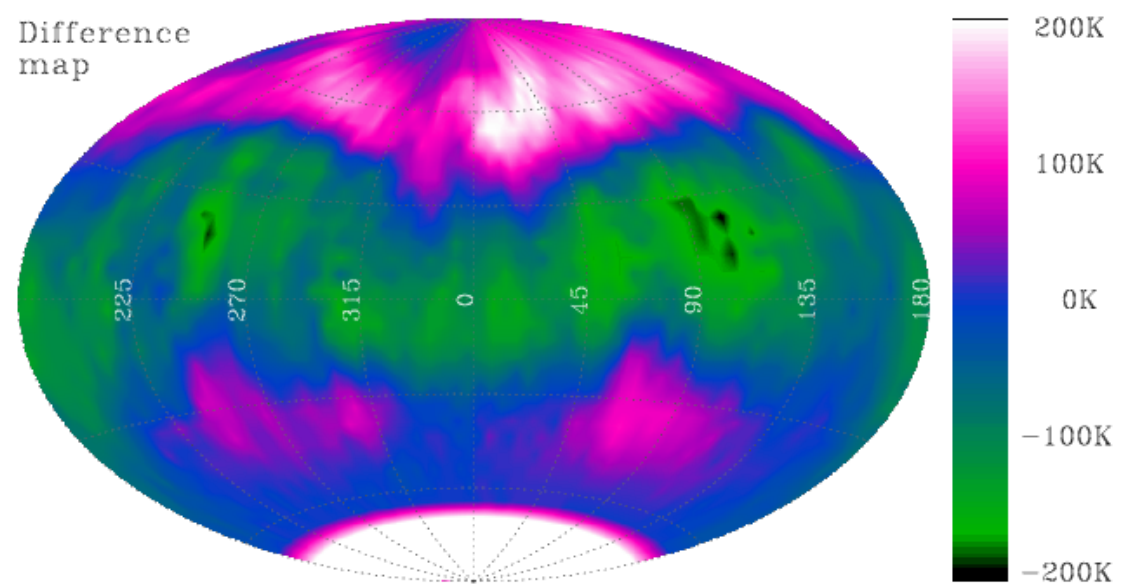

Figure 4. Top panel: Doppler image of $\zeta$ And using our nonspherical model with $\epsilon=0.27$ and $v \sin i=41.4 \mathrm{~km} \mathrm{~s}^{-1}$. Middle panel: resulting Doppler map using a simple spherical model $(\epsilon=0.0)$. Bottom: temperature difference map obtained by subtracting the nonspherical model (top) from the spherical one (middle). (Note that the distortion itself is not represented directly in the figure, thus the map of the distorted surface at the top and the sphere at the middle appear in a similar shape.) 
our case, the elliptical distortion $\epsilon$ of the primary. With this iterative imaging procedure we can redetermine the stellar parameters with a higher accuracy (see e.g., Unruh 1996; Weber \& Strassmeier 1998).

With the consideration in mind that the most closely connected values are $v \sin i$ and the distortion parameter $\epsilon$, we scan through a meaningful part of the parameter space. Changing $v \sin i$ and $\epsilon$ while all other parameters are held constant yields a certain variation in the $\chi^{2}$ of the line-profile fits. The resulting $\chi^{2}$ map is shown in Figure 3. The minimum corresponds to $\epsilon=0.27 \pm 0.03$ and $v \sin i=41.4 \pm 0.5 \mathrm{~km} \mathrm{~s}^{-1}$.

The resulting ellipticity parameter is in very good agreement with the result of our photometric study (see Kövári et al. 2006). Also the obtained $v \sin i$ is well within the range found by other authors (e.g., Fekel et al. 1999).

As the proof of the pudding the resulting astrophysical input parameters are used for Doppler imaging a series of Ca I-6439 spectra obtained at KPNO in 1996-1997. The Doppler map is shown in Figure 4 together with the Doppler image obtained assuming spherical shape and also a difference map. This latter is obtained by subtracting the original image (i.e., the nonspherical model) from the one assuming spherical shape. Similarity with the prediction (cf. Figure 2) is striking.

\section{Discussion}

When taking an ellipsoidal variable the distorted shape results in a slightly changing $v \sin i$ through the rotation phase, thus in our study $v \sin i$ means an average value: the rotational broadening is minimum at phases 0.0 and 0.5 when the 'cigar' is viewed from the 'back' and from the 'front' side (facing the secondary), respectively, while $v \sin i$ is maximum at 'side-on' views. When the 'cigar' is approximated with a sphere the replacing visible diameter is slightly extended at phases 0.0 and 0.5 , i.e. using the sphere results in $v \sin i$ getting a bit higher. This surplus, however, can be reduced when the two sides of the apparent disc are faded by darker areas. Compensating large cool patches centering at the quadratures appear similarly in the test figure (Figure 2) and in the real difference map (bottom panel of Figure 4).

For further discussions and other details on Doppler imaging $\zeta$ And see our forthcoming paper by Kővári et al. (2006).

\section{Acknowledgements}

ZsK is a grantee of the Bolyai János Scholarship. ZsK, KO and EF-D are very grateful to the Hungarian Science Research Program (OTKA) for support under grants OTKA T043504 (ZsK, KO) and OTKA-T048961 (ZsK, KO, EF-D). JR acknowledges the financial support of the National Sciences and Engineering Research Council of Canada. EF-D is grateful for support from the European Solar Magnetism Network, funded by the European Commission under contract HPRN-CT-2002-00313.

\section{References}

Hall, D.S. 1990, AJ 100, 554

Fekel, F.C., Strassmeier, K.G., Weber, M., \& Washuettl, A. 1999, A\&AS 137, 369

Kővári, Zs., Bartus, J., Strassmeier, K.G., Oláh, K., Rice, J.B., \& Csizmadia, Sz. 2005, Proc. Cool Stars, Stellar Systems and the Sun 13, 5-9 July 2004, Hamburg, Germany, ESA-SP-560 (Vol. II), 727

Kővári, Zs., Bartus, J., Strassmeier, K.G., Oláh, K., Weber, M., Rice, J.B., \& Washuettl, A. $2006 A \mathscr{E} A$ in press (preprint doi http://dx.doi.org/10.1051/0004-6361:20065982

Rice, J.B., Wehlau, W.H., \& Khokhlova, V.L. 1989, A\&SA 208, 179 
Strassmeier, K.G., Hall, D.S., Fekel, F.C., \& Scheck, M. 1993, A\&A 100, 173

Strassmeier, K.G., Hall, D.S., Boyd, L., \& Genet, R. 1989, ApJS 69, 141

Unruh, Y.C. 1996, Proc. IAU Symp. 176: Stellar Surface Structure, Strassmeier, K.G. ES Linsky, J.L., eds. 35

von Zeipel, H. 1924, MNRAS 84, 665

Weber, M., Strassmeier, K.G. 1998, A\&\&A 330, 1029 